\title{
Investigation of chemical phase formation in the ternary system beryllium, carbon and tungsten with depth-resolved photoelectron spectroscopy
}

\author{
F. Kost, Ch. Linsmeier, M. Oberkofler, M. Reinelt, M. Balden, A. Herrmann, S. Lindig \\ Max-Planck-Institut für Plasmaphysik, EURATOM Association, Boltzmannstrasse 2, 85748 \\ Garching b. München, Germany
}

\begin{abstract}
It is presented that synchrotron radiation XPS with different photon energies can be used to gain depth-resolved chemical information. The temperature-dependent evolution of a ternary system sample with beryllium, carbon and tungsten is investigated. Five temperature steps are performed in order to analyze the kinetic processes and chemical states, namely 300, 530, 850,1020 and $1200 \mathrm{~K}$. After each temperature treatment, the sample composition is analysed using four different information depths.
\end{abstract}

PACS: 52.40.Hf; 82.80.Pv; 41.60.Ap; 82.40.-g

JNM keywords: Beryllium, Carbon, Tungsten, X-ray techniques and applications, Chemical reactions

PSI-18 keywords: Beryllium, Carbide, First Wall Materials, Surface analysis, Diffusion corresponding author E-mail: florian.kost@ipp.mpg.de corresponding author address: Max-Planck-Institut für Plasmaphysik, EURATOM Association, Boltzmannstrasse 2, 85748 Garching b. München, Germany

Presenting author: Christian Linsmeier

Presenting author E-mail: linsmeier@ipp.mpg.de 


\section{Introduction}

For the future fusion experiment ITER, the three elements beryllium, carbon and tungsten are planned to be used as first wall materials [1]. The interaction between these materials, in particular the developing compounds due to temperature treatment, are of major interest as they strongly influence fusion relevant material properties such as erosion behaviour, melting point and hydrogen inventory. The knowledge of binary systems is well established, but the complexity increases considerably with the addition of a third component. In order to understand these interactions, model systems with thin elemental layers of a few nm thickness are prepared, annealed and analysed by X-ray photoelectron spectroscopy (XPS). For the characterization of temperature-driven processes such as diffusion, the XPS technique is applied using synchrotron radiation. By changing the excitation energy in the XPS process, it is possible to gain information from within different depths of the sample [2-6]. Compared to sputter depth profiling, this method has the advantage of being non-destructive and therefore, it does not alter the chemical composition of the investigated system. Hence, it allows an elemental and chemical state analysis as a function of depth.

\section{Experimental}

A carbon-beryllium-tungsten (C-Be-W) ternary system is prepared using physical vapour deposition. Polished polycrystalline tungsten is used as substrate material and carbon acts as a passivating layer on the top in order to minimise the influence of oxygen contaminations since it is less reactive towards oxidation than $\mathrm{W}$ and, particularly, Be. The beryllium layer is intermediate between the substrate and the carbon layer. The film thicknesses are $2.4 \mathrm{~nm}$ for the beryllium layer and $1.5 \mathrm{~nm}$ for the carbon layer. The sample is prepared under vacuum conditions ( $\mathrm{p}<8 \mathrm{E}-7 \mathrm{~Pa}$ during evaporation) and then transferred to the synchrotron beamline via transport through air.

XPS measurements are performed using a hemispherical electron analyzer at the MUSTANG endstation at the RGBL-PGM of the synchrotron facility BESSY in Berlin. The angle between the incident X-ray and the photoelectron take-off direction is $45^{\circ}$. For all the measurements, the photoelectron take-off direction is normal to the sample surface and the pass energy is $2 \mathrm{eV}$. The regions to measure are $\mathrm{Be} 1 \mathrm{~s}, \mathrm{C} 1 \mathrm{~s}$ and $\mathrm{W} 4 \mathrm{f}$.

The sample is heated up to different temperatures and the XPS measurements are done stepwise after each temperature treatment of 300, 530, 850, 1020 and $1200 \mathrm{~K}$. The annealing time is 30 minutes for each step. In the photo-ionisation process, the photoelectron kinetic 
energy is determined by the incident photon excitation energy and the core level of the element emitting the photoelectrons (e.g. Be 1s, $\mathrm{C} 1 \mathrm{~s}$ and $\mathrm{W} 4 \mathrm{f}$ in this work). As the kinetic energy of the photoelectrons strongly influences the inelastic mean free path [7], the excitation energy is chosen such that the photoelectrons originate from within the same depths, i.e. the inelastic mean free path is equal for different elements. Four different information depths are chosen for analysis.

For each spectrum, the binding energies are calibrated by measuring the Au $4 \mathrm{f}$ signal. Assuming a homogenous material, the intensity $I\left(E_{\mathrm{k}}, x\right)$ for a photoelectron peak of kinetic energy $E_{\mathrm{k}}$ excited from the level $x$ is given by $I\left(E_{\mathrm{k}}, x\right)=J(h v) \cdot N_{\mathrm{i}} \cdot \sigma(h v, \theta, x) \cdot \lambda\left(E_{\mathrm{k}}\right) \cdot T\left(E_{\mathrm{k}}\right)$, where $J(h v)$ is the photon flux incident on the sample at the energy $h v, N_{\mathrm{i}}$ is the density of atoms of the $i$ th species, $\sigma(h v, \theta, x)$ is the photoelectron cross section for the level $x$ of the $i$ th species at energy $h v$ and electron ejection angle $\theta ; \lambda\left(E_{\mathrm{k}}\right)$ is the inelastic mean free path in the sample for electrons of kinetic energy $E_{\mathrm{k}}$ and $T\left(E_{\mathrm{k}}\right)$ is the transmission function of the analyzer. The photon flux and the analyzer transmission are defined by the experimental setup. The photoelectron cross section $\sigma(h v, \theta, x)$ is separated into an angle-independent part and an angledependent asymmetry factor $L, \sigma(h v, \theta, x)=\sigma(h v, x) \cdot L(h v, \theta, x)$. For the polarized light used at the synchrotron beamline, the asymmetry factor is $L(h v, \theta, x)=1+0.5 \beta\left(3 \cos ^{2} \theta-2\right)$, where $\beta$ is the asymmetry parameter [8]. For quantitative analysis, the peak areas are normalized against photoionisation cross-section [8], asymmetry parameter [9], ring current, photon flux and analyzer transmission.

Since one element is measured with different photon energies, the electrons have different inelastic mean free paths $\lambda$. Hence, taking into account Lambert-Beer's Law $I=I_{0} \cdot \exp (-d /(\lambda \cdot \cos \theta))$, absolute peak areas from the same depths $d$ are not comparable. Therefore, relative signal intensities are considered, which are normalized against the total signal intensity, which is the sum over all contributions. As the exact information depths for the photoelectrons cannot be determined, it is necessary to refer to the different depths as $d l$ (near-surface region) to $d 4$ (largest information depth). Taking into account the mean free path dependence on photon energy, the chosen photoelectron kinetic energies are $60 \mathrm{eV}$ (nearsurface region), $300 \mathrm{eV}, 480 \mathrm{eV}$ and $700 \mathrm{eV}$ (largest information depth). Since relative signal intensities are normalized against the sum over all contributions, it is possible to compare relative signal intensities of different depth regions (even though the inelastic mean free path changes from $d 1$ to $d 4$ ). 


\section{Results and discussions}

Figure 1 shows the $\mathrm{C} 1 \mathrm{~s}$ signal after normalisation. Figure 1a shows measurements with a photoelectron kinetic energy of $60 \mathrm{eV}$, while the measurements with a kinetic energy of $700 \mathrm{eV}$ are shown in Fig 1b. The C 1s peak shape is fitted by background subtraction (Shirleybackground, Ref [10]) and three Gauss-Lorentzian peaks: Elemental carbon is described by a graphitic $\left(E_{\mathrm{B}}=284.2 \mathrm{eV}\right)$ and a disordered graphitic $\left(E_{\mathrm{B}}=285.1 \mathrm{eV}\right)$ contribution. The beryllium carbide peak is clearly visible in the binding energy region around $282 \mathrm{eV}$.

In the near-surface region (a), the carbide signal is not observed up to $850 \mathrm{~K}$. At $1020 \mathrm{~K}$, a small amount of $\mathrm{Be}_{2} \mathrm{C}$ is detected. For the greater information depth (b), a certain amount of beryllium carbide is observed at room temperature. The carbide intensity rises during the temperature treatment and is most pronounced at $1020 \mathrm{~K}$. At the last temperature, $1200 \mathrm{~K}$, the beryllium carbide peak slightly shifts to lower binding energies. This binding energy shift might be due to the growth of $\mathrm{Be}_{2} \mathrm{C}$ clusters and following cluster size effect. The cluster size is known to have an important influence on the binding energy [11]. The slight shift in the binding energy and peak shoulders at $\sim 287 \mathrm{eV}$ of the 300 and $580 \mathrm{~K}$ elemental carbon peaks indicate a surface contamination due to transport through air. However, this contribution is taken into account during the fitting procedure, but disregarded in the sample composition analysis.

The room temperature measurements are chosen to demonstrate the analysis of XPS spectra with different photon energies. The region $d l$ (photoelectron kinetic energy $60 \mathrm{eV}$ ) shows $\mathrm{BeO}$ with a relative signal intensity of $22 \%$ and elemental carbon with $78 \%$. After sample preparation, no beryllium was present on the surface. This points to a Be diffusion (driven by the chemical gradient) to the surface where oxygen was available due to the transport through air.

The next information depth $d 2$ (photoelectron kinetic energy $300 \mathrm{eV}$ ) shows $2 \%$ of metallic beryllium, $16 \%$ of $\mathrm{BeO}$ and $82 \%$ of elemental carbon. We want to emphasize here, that the increasing kinetic energy of the photoelectrons increases the information depth, thus adding information of a greater depth to the overall measured relative elemental composition. Taking into account the exponential decay of the photoelectron intensity according to Lambert-Beer's Law, the contribution from surface components is less influential as the information depth increases. Regarding the decreasing relative intensity of $\mathrm{BeO}$ for $d 2$ from $22 \%$ to $16 \%$, there is less $\mathrm{BeO}$ present in deeper regions. Vice versa, more elemental carbon is detected 
compared to $d 1$. The relative signal intensities for $d 3$ (photoelectron kinetic energy $480 \mathrm{eV}$ ) are $12 \%$ metallic $\mathrm{Be}, 3 \%$ beryllium carbide $\left(\mathrm{Be}_{2} \mathrm{C}\right), 23 \% \mathrm{BeO}$ and $62 \%$ elemental $\mathrm{C}$. As there is a distinct contribution from metallic beryllium, the information depth now clearly exceeds the carbon overlayer. Both the $\mathrm{Be}$ amount and the $\mathrm{BeO}$ amount increase for $d 3$. As the relative signal intensity of $\mathrm{BeO}$ increases in measurement $d 3$ compared to $d 2$, this points not only to $\mathrm{BeO}$ on the surface but also to $\mathrm{BeO}$ in the metallic $\mathrm{Be}$ layer (below carbon). A small amount of $\mathrm{Be}_{2} \mathrm{C}$ is detected at this information depth. Hence, the carbide formation at room temperature starts at the $\mathrm{Be} / \mathrm{C}$ interface. $\mathrm{Be}_{2} \mathrm{C}$ formation at room temperature is well known and has been investigated in Ref [12]. The relative signal intensities for $d 4$ (photoelectron kinetic energy $700 \mathrm{eV}$ ) are $18 \%$ metallic $\mathrm{Be}, 4 \% \mathrm{Be}_{2} \mathrm{C}, 25 \% \mathrm{BeO}, 50 \%$ elemental carbon and $2 \%$ elemental tungsten. Since the relative intensities for the beryllium components increase, there is more $\mathrm{Be}, \mathrm{Be}_{2} \mathrm{C}$ and $\mathrm{BeO}$ visible in $d 4$. As there is a contribution from tungsten for $d 4$, this information depth exceeds both the beryllium and carbon overlayers. The relative signal intensity of tungsten is not crucial regarding the total sample composition but nevertheless, metallic tungsten and $\mathrm{Be}_{2} \mathrm{~W}$ alloy are present.

The first annealing temperature is $530 \mathrm{~K}$. The $\mathrm{C} 1 \mathrm{~s}$ signal is taken as an example in Fig. 2 to point out the sample composition development during temperature treatment. The relative signal intensity of elemental carbon decreases from room temperature to the third annealing step, $1020 \mathrm{~K}$. The relative signal intensity is not maximal for $d 1$, but for $d 2$. This is due to the $\mathrm{BeO}$ overlayer, which is discussed above. As $\mathrm{BeO}$ is very prominent at the surface, the relative signal intensity of elemental carbon is smaller. Furthermore, the relative intensity of elemental carbon decreases from $d 2$ to $d 4$, since other contributions such as metallic Be and $\mathrm{Be}_{2} \mathrm{C}$ become more prominent in greater depths. At the last temperature step, $1200 \mathrm{~K}$, the relative signal intensity for elemental carbon is very distinct at the surface and decreases with increasing information depth. This might be due to carbon diffusion to the surface or a change in surface morphology, such as formation of island-like structures.

The relative signal intensity of $\mathrm{Be}_{2} \mathrm{C}$ increases from $d l$ to $d 4$. This indicates a beginning growth of beryllium carbide from the $\mathrm{Be} / \mathrm{C}$ interface and expanding to the surface. At the last temperature step, $1200 \mathrm{~K}$, the overall relative signal intensity of $\mathrm{Be}_{2} \mathrm{C}$ decreases. Since relative signal intensities are considered, the relative intensity of $\mathrm{Be}_{2} \mathrm{C}$ is connected with that of elemental C. 
At $530 \mathrm{~K}$, the relative signal intensity of $\mathrm{Be}_{2} \mathrm{C}$ increases for $d 3$ and $d 4$ and furthermore, $\mathrm{Be}_{2} \mathrm{C}$ is detected in $d 2$. This is another hint to beryllium carbide growth from the interface to the surface. According to an increasing $\mathrm{Be}_{2} \mathrm{C}$ amount, the amounts of metallic $\mathrm{Be}$ and elemental carbon decrease. The amount of $\mathrm{BeO}$ at the surface is increasing due to rising pressure during temperature treatment, which gives rise to further oxide formation. Like for room temperature, a small contribution from tungsten is detected for $d 4$ and the amount of the $\mathrm{Be}_{2} \mathrm{~W}$ alloy is increasing.

At $850 \mathrm{~K}$, the relative intensity of $\mathrm{Be}_{2} \mathrm{C}$ increases for $d 2$ to $d 4$ and furthermore, $\mathrm{Be}_{2} \mathrm{C}$ is detected in the near-surface region $d 1$, giving another hint for carbide growth from the interface to the surface. The amount of $\mathrm{BeO}$ at the surface is increasing further and no metallic $\mathrm{Be}$ is left at this temperature step. As more $\mathrm{Be}_{2} \mathrm{C}$ is forming, the total amount of elemental carbon decreases. At $850 \mathrm{~K}$, the amount of $\mathrm{Be}_{2} \mathrm{~W}$ is decreasing, whereas an onset of $\mathrm{W}_{2} \mathrm{C}$ formation is observed,

At $1020 \mathrm{~K}$, the relative intensity of $\mathrm{Be}_{2} \mathrm{C}$ increases for $d 1$ to $d 4$. Furthermore, the amount of $\mathrm{BeO}$ at the surface increases. As no metallic $\mathrm{Be}$ is available to form additional $\mathrm{Be}_{2} \mathrm{C}$ or $\mathrm{BeO}$, this increase must be due to a change in surface morphology, thus giving more weight to nearsurface components. According to the increase of $\mathrm{Be}_{2} \mathrm{C}$ and $\mathrm{BeO}$, the relative intensity of elemental carbon decreases for all depths. The relative signal intensity of tungsten is still not crucial regarding the total sample composition, but the amount of the $\mathrm{Be}_{2} \mathrm{~W}$ alloy is decreasing further, whereas the amount of the carbide $\mathrm{W}_{2} \mathrm{C}$ increases.

At $1200 \mathrm{~K}$, the relative intensities of $\mathrm{Be}_{2} \mathrm{C}$ and $\mathrm{BeO}$ decrease for $d 1$ to $d 4$, whereas the relative intensity of elemental carbon increases. As discussed for Fig. 2, this might be due to carbon diffusion to the surface or a change in surface morphology, such as formation of island-like structures. Furthermore, metallic tungsten and tungsten carbide $\mathrm{W}_{2} \mathrm{C}$ is detected in the near-surface region $d 1$. If tungsten diffusion was assumed, a carbide or alloy phase at the surface would be expected. The presence of metallic tungsten might be ascribed to the appearing substrate which gives hint to a formation of island-like structures. At $1200 \mathrm{~K}$, the $\mathrm{Be}_{2} \mathrm{~W}$ alloy has completely vanished. The tungsten signal is composed of metallic and carbidic $\left(\mathrm{W}_{2} \mathrm{C}\right)$ tungsten.

The results from the system $\mathrm{Be} / \mathrm{C} / \mathrm{W}$ are consistent with the analysis of binary systems. In experiments with carbon layers on a beryllium substrate, beryllium carbide growth from the interface to the surface is observed [13]. At $770 \mathrm{~K}, \mathrm{Be}_{2} \mathrm{C}$ formation is complete [12]. Experiments with beryllium layers of a few nm thickness on a pyrolytic graphite substrate 
show island formation at $1070 \mathrm{~K}$. In the ternary system, beginning formation of island-like structures is observed at $1020 \mathrm{~K}$. Experiments with carbon layers on tungsten show C diffusion into the bulk material and $\mathrm{W}_{2} \mathrm{C}$ formation starting at $870 \mathrm{~K}$. Alloy formation at room temperature is also observed in the binary system $\mathrm{Be} / \mathrm{W}$.

\section{Summary}

A layered system of beryllium, carbon and tungsten is prepared, annealed and analysed by depth-resolved photoelectron spectroscopy. With this technique, it is feasible to characterize reactions by means of depth-resolved information. Beryllium diffusion through carbon to the surface and $\mathrm{BeO}$ formation is observed at room temperature. The beryllium carbide formation starts at the interface and expands to the surface. It is complete at $850 \mathrm{~K}$. Carbon diffuses into the bulk and starts forming $\mathrm{W}_{2} \mathrm{C}$ at $850 \mathrm{~K}$. At $1020 \mathrm{~K}$, the analysis gives hint to a change in surface morphology. This effect is even more pronounced at $1200 \mathrm{~K}$. The formation of a $\mathrm{Be}_{2} \mathrm{~W}$ alloy is observed at room temperature. The alloy amount increases at $530 \mathrm{~K}$; as the temperature rises to $850 \mathrm{~K}$, the alloy amount is decreasing, whereas the amount of $\mathrm{W}_{2} \mathrm{C}$ increases. The amount of tungsten carbide increases up to the last temperature $1200 \mathrm{~K}$.

\section{Acknowledgements}

We thank Gianina Gavrialla, Maria Brzhezkinskaya and Mike Sperling for the support at the synchrotron beamline. 


\section{References}

1 Federici, G., Anderl, R., Brooks, J. N., Causey, R., Coad, J. P., Cowgill, D., Doerner, R., Haasz, A. A., Longhurst, G., Luckhardt, S., Mueller, D., Peacock, A., Pick, M., Skinner, C. H., Wampler, W., Wilson, K., Wong, C., Wu, C., Youchison, D., Fusion Engineering and Design 39-40 (1998) 445.

2 Esaka, F., Furuya, K., Shimada, H., Imamura, M., Matsubayashi, N., Sato, T., Nishijima, A., Kikuchi, T., Kawana, A., Ichimura, H., Surface Science 377-379 (1997) 197.

3 N. Matsubayashi, Singh, B. P., Imamura, M., Tanaka, T., Sato, Y., Ogiwara, T., Suzuki, M., Kiyota, S., Surface and Interface Analysis 36 (2004) 853.

4 Shimada, H., Matsubayashi, N., Imamura, M., Sato, T., Nishijima, A., Applied Surface Science 100-101 (1996) 56.

5 Shimada, H., Sato, K., Matsubayashi, N., Imamura, M., Saito, T., Furuya, K., Applied Surface Science 144-145 (1999) 21.

6 Zier, M., Oswald, S., Reiche, R., Wetzig, K., Microchimica Acta 156 (2006) 99.

$7 \quad$ M. P. Seah, W. A. D., Surface and Interface Analysis 1 (1979) 2.

$8 \quad$ Yeh, J. J., Lindau, I., Atomic Data and Nuclear Data Tables 32 (1985) 1.

$9 \quad$ M. P. Seah, I. S. G., Surface and Interface Analysis 31 (2001) 835.

10 Shirley, D. A., Phys. Rev. B 5 (1972) 4709.

11 Mason, M. G., Physical Review B 27 (1983) 748.

12 Goldstrass, P., Klages, K. U., Linsmeier, C., J. Nucl. Mater. 290-293 (2001) 76.

13 Roth, J., Wampler, W. R., Jacob, W., J. Nucl. Mater. 250 (1997) 23. 


\section{Figure captions}

Figure 1:

XPS C 1s signal for $1.5 \mathrm{~nm}$ carbon and $2.4 \mathrm{~nm}$ beryllium layers evaporated on a tungsten substrate. The temperature steps are indicated on the right. The upper panel (a) shows measurements with a photoelectron kinetic energy of $60 \mathrm{eV}$ (near-surface region), while the lower panel shows data with a kinetic energy of $700 \mathrm{eV}$ (largest information depth). The beryllium carbide peak is developing at $\sim 282 \mathrm{eV}$ and expanding from the interface to the surface.

Figure 2:

Relative signal intensity of elemental carbon (solid symbols) and beryllium carbide (open symbols) as a function of temperature. The symbol shape indicates the photoelectron kinetic energy as indicated on the right. The amount of beryllium carbide increases with increasing information depth. The change at $1200 \mathrm{~K}$ is due to a change in the surface morphology. 
Figures

Figure 1

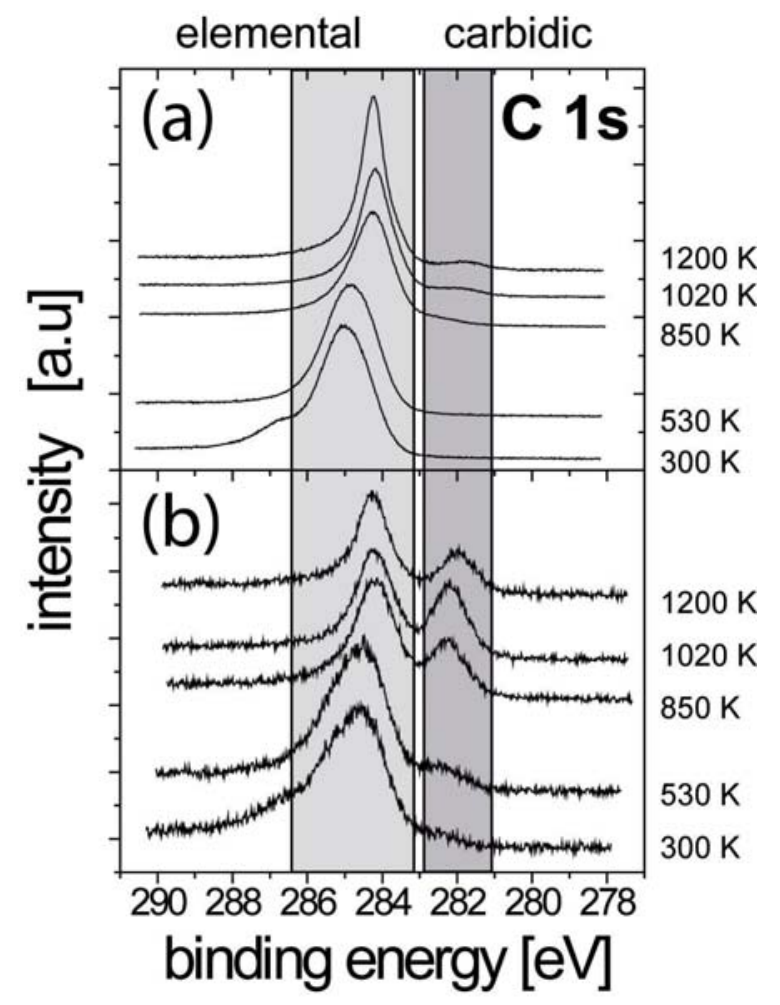

Figure 2

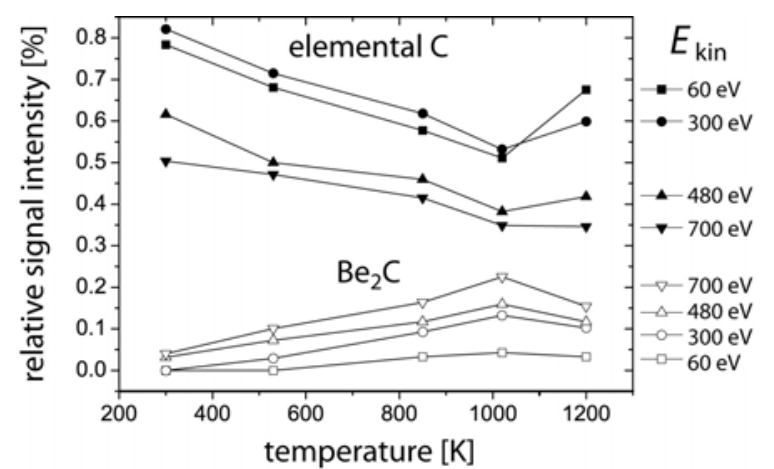


